\title{
Stochastic Small Signal Interval Stability of Power Systems with Asynchronous Wind Turbine Generators
}

\author{
Zhen Zhang and Yanhong Liu \\ School of Electrical Engineering, Zhengzhou University, Zhengzhou 450001, China \\ Correspondence should be addressed to Yanhong Liu; liuyh@zzu.edu.cn
}

Received 13 August 2017; Accepted 26 November 2017; Published 11 January 2018

Academic Editor: Quanxin Zhu

Copyright (c) 2018 Zhen Zhang and Yanhong Liu. This is an open access article distributed under the Creative Commons Attribution License, which permits unrestricted use, distribution, and reproduction in any medium, provided the original work is properly cited.

\begin{abstract}
The stochastic dynamic interval model of power systems with asynchronous wind turbine generators is established with consideration of the interval uncertain parameters and random small excitation disturbances. The conditions of interval mean stability and interval mean square stability of the power systems are proposed. The relationship between the bounds of the mean (mean square) error and the parameter interval range of the systems is discussed. Finally, we simulate the power systems to demonstrate the effectiveness of the proposed results.
\end{abstract}

\section{Introduction}

With the rapid development of wind power and other emerging new energy and grid, the random excitation has a significant impact on the power systems stability and power quality [1-3]. In addition, the system parameters are generally uncertain because of the effects of the temperature, humidity, and other environmental conditions and the restriction of the information collection technology. It is of great importance to consider the influence of the stochastic excitation and uncertain parameters in the stability analysis of power systems [4$6]$.

In recent years, a lot of researches have been done on the stability analysis and feedback control of stochastic systems; see [7-10] and the references therein. For power systems under stochastic excitations, Humberto et al. [11] discussed the almost sure asymptotic stability of linear stochastic systems with bounded Markov diffusion process perturbation by the means of Lyapunov exponents and applied the theoretical results to one machine infinite bus electric power systems. Ma et al. established a continuous Markov power system model with multiple operating conditions considering the stochastic characteristic of wind speed and proposed a Lyapunov function based method for the stability analysis of the system [4]. Robust stochastic stability of power systems under stochastic excitation and random perturbations was discussed in $[12,13]$. Noticing that the mean and mean square stability $[14,15]$ are of great importance for power systems, the small signal mean stability and mean square stability of power systems under random excitation were investigated by some researchers. In [2], Zhang et al. put forward a stochastic differential equation model for power system under Gauss type random excitation and proposed some conditions for the mean stability and mean square stability of the system. Yuan et al. further investigated the the steady-state expectation and covariance of the system state variables of the power systems with asynchronous wind turbines [16]. For a general case of mean stability and mean square stability, Lu et al. discussed the $p$ th stochastic stability and dynamic characteristics of power systems under small Gauss type random excitations [17]. For power systems whose network parameters are being known to be within certain bounds, under the assumption of with mixed phasor and conventional power measurements, some state estimation methods were put forward based on the interval linear system model $[18,19]$. So far, there have been no researches carried out for the stochastic mean stability and mean square stability analysis of power systems with both consideration of random excitation disturbances and interval parameter uncertainties.

In this paper, we discuss the stochastic small signal stability of power systems with wind turbines and parameter uncertainties. First, the stochastic interval dynamic model of 


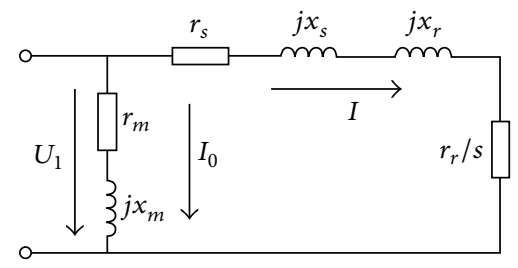

FIGURE 1: The simplified equivalent circuit of the asynchronous generators.

the power systems is established with consideration of the interval parameter uncertainties and small random excitations. Then, we propose some results for the mean stability and mean square stability by analyzing the eigenvalues of the system matrix. The relationship between the bounds of the mean and the mean square error and the small random excitation and interval uncertain parameters is also discussed. Finally, we simulate a single machine infinite bus power system with asynchronous wind turbine Generator to verify the effectiveness of the proposed method.

The paper is organized as follows. First, the stochastic dynamic interval model of power system is established considering interval uncertain parameters under small random excitation in Section 2. The interval mean stability and interval mean square stability of the system are given in Section 3. Section 4 gives the simulation of power systems. Finally, conclusions are drawn in the last section.

\section{Stochastic Dynamic Model of Power Systems with Asynchronous Wind Turbine Generators}

2.1. Dynamic Model of Asynchronous Generators. Ignoring the electromagnetic transient process of asynchronous generator, the simplified equivalent circuit of the asynchronous generator can be shown in Figure 1 [20], where $r_{s}, x_{s}, r_{r}$, $x_{r}, r_{m}, x_{m}$, and $U_{1}$ are the stator resistance, the stator reactance, the rotor resistance, the rotor reactance, the excitation resistance, the excitation reactance, and the voltage of the asynchronous generator, respectively, $s$ is the slip, and $I$ is the stator current.

From Figure 1, we have

$$
I^{2}=\frac{U_{1}^{2}}{\left(r_{s}+r_{r} / s\right)^{2}+\left(x_{s}+x_{r}\right)^{2}} .
$$

The active power consumption in the rotor windings can be formulated as

$$
\Delta P_{E}=\frac{U_{1}^{2} r_{r}}{\left(r_{s}+r_{r} / s\right)^{2}+\left(x_{s}+x_{r}\right)^{2}} .
$$

The electromagnetic power and the mechanical power of the system are

$$
P_{E}=\frac{U_{1}^{2} r_{m}}{r_{m}^{2}+x_{m}^{2}}+\frac{U_{1}^{2}\left(r_{s}+r_{r} / s\right)}{\left(r_{s}+r_{r} / s\right)^{2}+\left(x_{s}+x_{r}\right)^{2}},
$$

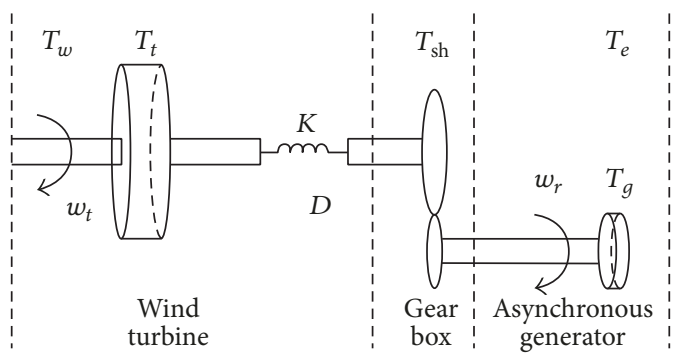

FIGURE 2: The mass model of wind turbine with asynchronous generators.

$$
P_{e}=\Delta P_{E}+P_{E}=\frac{U_{1}^{2} r_{m}}{r_{m}^{2}+x_{m}^{2}}+\frac{U_{1}^{2}\left(r_{r}+r_{s}+r_{r} / s\right)}{\left(r_{s}+r_{r} / s\right)^{2}+\left(x_{s}+x_{r}\right)^{2}},
$$

respectively. Writing the parameters ware as per unit values, we have $T_{e}=P_{e}$ and

$$
T_{e}=\frac{U_{1}^{2} r_{m}}{r_{m}^{2}+x_{m}^{2}}+\frac{U_{1}^{2}\left(r_{r}+r_{s}+r_{r} / s\right)}{\left(r_{s}+r_{r} / s\right)^{2}+\left(x_{s}+x_{r}\right)^{2}} .
$$

Ignoring the high-end items, the Taylor series expansion of $T_{e}$ at $s_{0}$ is

$$
T_{e}=T_{e 0}+\frac{d T_{e}}{d s} \Delta s
$$

Assuming $R=d T_{e} / d s$, we have

$$
\begin{aligned}
R=- & \frac{U_{1}^{2} r_{r}\left[\left(r_{s}+r_{r} / s\right)^{2}+\left(x_{s}+x_{r}\right)^{2}\right]}{s^{2}\left[\left(r_{s}+r_{r} / s\right)^{2}+\left(x_{s}+x_{r}\right)^{2}\right]^{2}} \\
& +\left.\frac{2 U_{1}^{2} r_{r}\left(r_{r}+r_{s}+r_{r} / s\right)\left(r_{s}+r_{r} / s\right)}{s^{2}\left[\left(r_{s}+r_{r} / s\right)^{2}+\left(x_{s}+x_{r}\right)^{2}\right]^{2}}\right|_{s=s_{0}} .
\end{aligned}
$$

2.2. Stochastic Model of Asynchronous Wind Turbine Generators. The asynchronous wind power generation systems is mainly composed of the wind turbine, gear box, and asynchronous generator, as shown in Figure 2.

Without consideration of the random excitation, the determinate shaft model of the wind turbines can be written as

$$
\begin{aligned}
T_{t} \frac{d \omega_{t}}{d t} & =T_{w}-T_{\mathrm{sh}}, \\
T_{g} \frac{d \omega_{r}}{d t} & =T_{\mathrm{sh}}-T_{e}, \\
\frac{d \theta_{w}}{d t} & =\left(\omega_{t}-\omega_{r}\right) \omega_{0},
\end{aligned}
$$

with

$$
T_{\mathrm{sh}}=K \theta_{w}+D \frac{d \theta_{w}}{d t}
$$


where $\omega_{t}$ is the angle speed of the wind turbine, $\omega_{0}$ is the synchronous angle speed, $\omega_{r}$ is the angle speed of the asynchronous generator, $T_{t}$ is the rotary inertia of the wind turbine, $T_{g}$ is the rotary inertia of the asynchronous generator, $T_{w}, T_{\mathrm{sh}}, T_{e}$ are output mechanical torque, shaft torque, and generator electromagnetic torque of wind turbine, respectively, $\theta_{w}$ is the shaft twist angle, $K$ is the shaft stiffness coefficient, and $D$ is the damping coefficient.

Considering the power fluctuations of the wind turbine and the asynchronous generator, which can be regarded as Gaussian random small excitation in a short time $[9,10]$, the stochastic dynamic model of the asynchronous wind turbine generators can be formulated as

$$
\begin{aligned}
T_{t} \frac{d \omega_{t}}{d t} & =T_{w}-T_{\mathrm{sh}}+\sigma_{1} W(t), \\
T_{g} \frac{d \omega_{r}}{d t} & =T_{\mathrm{sh}}-T_{e}+\sigma_{2} W(t), \\
\frac{d \theta_{w}}{d t} & =\left(\omega_{t}-\omega_{r}\right) \omega_{0},
\end{aligned}
$$

where $\sigma_{1}$ and $\sigma_{2}$ are the random excitation intensities and $W(t)$ is the standard Wiener process.

Substituting (9) into (10), we have the stochastic dynamic model of the asynchronous wind turbine generators as follows:

$$
\begin{aligned}
& T_{t} \frac{d \omega_{t}}{d t}=T_{w}-K \theta_{w}-D\left(\omega_{t}-\omega_{r}\right) \omega_{0}+\sigma_{1} W(t), \\
& T_{g} \frac{d \omega_{r}}{d t}=K \theta_{w}+D\left(\omega_{t}-\omega_{r}\right) \omega_{0}-T_{e}+\sigma_{2} W(t) .
\end{aligned}
$$

2.3. Stochastic Interval Model of Power Systems with Wind Turbines. In this section, the stochastic dynamic interval model of power systems with asynchronous wind turbine generators is established with consideration of the parameter uncertainty of $D$.

Considering the random small excitation and denoting $\omega_{t}=\omega_{0}+\Delta \omega_{t}, \omega_{r}=\omega_{0}+\Delta \omega_{r}$, we have

$$
\begin{aligned}
\frac{d \Delta \omega_{t}}{d t}= & \frac{1}{T_{t}}\left[T_{w}-K \theta_{w}-D\left(\Delta \omega_{t}-\Delta \omega_{r}\right) \omega_{0}\right] \\
& +\frac{\sigma_{1}}{T_{t}} W(t), \\
\frac{d \Delta \omega_{r}}{d t}= & \frac{1}{T_{g}}\left[K \theta_{w}+D\left(\Delta \omega_{t}-\Delta \omega_{r}\right) \omega_{0}-T_{e}\right] \\
& +\frac{\sigma_{2}}{T_{g}} W(t) .
\end{aligned}
$$

Noting that $\omega_{r}=(1-s) \omega_{0}$ and $\Delta \omega_{r}=-s \omega_{0}$, we can obtain

$$
\begin{aligned}
\frac{d \Delta \omega_{t}}{d t}= & \frac{1}{T_{t}}\left[T_{w}-K \theta_{w}-D\left(\Delta \omega_{t}+s\right) \omega_{0}\right]+\frac{\sigma_{1}}{T_{t}} W(t), \\
\frac{d s}{d t}= & -\frac{1}{T_{g} \omega_{0}}\left[K \theta_{w}+D\left(\Delta \omega_{t}+s\right) \omega_{0}-T_{e}\right] \\
& -\frac{\sigma_{2}}{T_{g} \omega_{0}} W(t) .
\end{aligned}
$$

When the asynchronous wind turbine generators are working at a stable operating point, we have

$$
T_{w}=K \theta_{w}=T_{e 0} .
$$

Let $s=s_{0}+\Delta s$; we can obtain

$$
\begin{aligned}
\frac{d \Delta \omega_{t}}{d t}= & -\frac{D \omega_{0}}{T_{t}} \Delta \omega_{t}-\frac{D \omega_{0}}{T_{t}} \Delta s-\frac{D \omega_{0}}{T_{t}} s_{0}+\frac{\sigma_{1}}{T_{t}} W(t), \\
\frac{d \Delta s}{d t}= & -\frac{D}{T_{g}} \Delta \omega_{t}-\frac{D \omega_{0}-R}{T_{g} \omega_{0}} \Delta s-\frac{D}{T_{g}} s_{0} \\
& -\frac{\sigma_{2}}{T_{g} \omega_{0}} W(t) .
\end{aligned}
$$

So the stochastic state equation of power systems with asynchronous wind turbine generators can be written as

$$
d x(t)=A x(t) d t+G d t+A^{\prime} d B(t),
$$

where $d B(t)=W(t) d t, x(t)=\left[\begin{array}{ll}\Delta \omega_{t} & \Delta s\end{array}\right]^{T}$,

$$
\begin{aligned}
& A=\left[\begin{array}{cc}
-\frac{D \omega_{0}}{T_{t}} & -\frac{D \omega_{0}}{T_{t}} \\
-\frac{D}{T_{g}} & -\frac{D \omega_{0}-R}{T_{g} \omega_{0}}
\end{array}\right], \\
& G=\left[\begin{array}{c}
-\frac{D \omega_{0}}{T_{t}} s_{0} \\
-\frac{D}{T_{g}} s_{0}
\end{array}\right], \\
& A^{\prime}=\left[\begin{array}{c}
\frac{\sigma_{1}}{T_{t}} \\
-\frac{\sigma_{2}}{T_{g} \omega_{0}}
\end{array}\right] .
\end{aligned}
$$


For power systems, the damping coefficient $D$ is always uncertain with some upper and lower bound. Denoting $D \in$ $[\underline{D}, \bar{D}]$, where $\underline{D}$ is the lower bound and $\bar{D}$ is the upper bound of $D$, respectively, we have $A \in[\underline{A}, \bar{A}]$, where

$$
\begin{gathered}
\underline{A}=\left[\begin{array}{cc}
-\frac{\bar{D} \omega_{0}}{T_{t}} & -\frac{\bar{D} \omega_{0}}{T_{t}} \\
-\frac{\bar{D}}{T_{g}} & -\frac{\bar{D} \omega_{0}-R}{T_{g} \omega_{0}}
\end{array}\right], \\
\bar{A}=\left[\begin{array}{cc}
-\frac{D \omega_{0}}{T_{t}} & -\frac{D}{T_{t}} \\
-\frac{D}{T_{g}} & -\frac{D}{T_{0}-R} \\
T_{g} \omega_{0}
\end{array}\right] .
\end{gathered}
$$

Let

$$
\begin{aligned}
A^{*} & =\frac{1}{2}(\underline{A}+\bar{A}), \\
\widetilde{A} & =\frac{1}{2}(\bar{A}-\underline{A}),
\end{aligned}
$$

so we have $A=A^{*}+\Delta A$ and $\Delta A \in(-\widetilde{A}, \widetilde{A})$. The stochastic dynamic interval model of the power systems with asynchronous wind turbine generators can be formulated as

$$
d x(t)=\left(A^{*}+\Delta A\right) x(t) d t+G d t+A^{\prime} d B(t) .
$$

\section{Stochastic Small Signal Interval Stability Analysis of the Power Systems}

In this section, we analyze the interval mean stability and interval mean square stability of the power systems and establish the relationship between the bounds of the mean and the mean square error and the system uncertain parameter and random excitation.

Definition 1 (see [8]). System (20) is interval p-moment stable, if

$$
\lim _{t \rightarrow \infty} E\|x(t)\|^{p}<\varepsilon
$$

where $\varepsilon>0$ is a constant. Especially, when $p=1$ and $p=2$, the system is interval mean stable and interval mean square stable, respectively.

Lemma 2 (see [21]). Let $Z$ be $n \times n$ real symmetric matrix and $x$ be n-dimensional column vector; we have

$$
\lambda_{\min }(Z) x^{T} x \leq x^{T} Z x \leq \lambda_{\max }(Z) x^{T} x
$$

where $\lambda_{\text {max }}(Z)$ and $\lambda_{\text {min }}(Z)$ are the maximum and minimum eigenvalue of $Z$, respectively.
Lemma 3 (see [22]). Consider the system

$$
d x(t)=f(x, t) d t
$$

If there exists a continuous positive definite function $V$ with infinitesimal upper bound and infinity lower bound such that

$$
L V=\frac{\partial V}{\partial t}+\sum_{i=1}^{n} f_{i}(x, t) \frac{\partial V}{\partial x_{i}}<0
$$

system (23) is asymptotically stable.

Theorem 4. Suppose there exists a reversible matrix $Q$ such that $P=Q J Q^{-1}$; we have

$$
\begin{aligned}
& \left\|e^{P}\right\| \leq\|Q\|\left\|Q^{-1}\right\| \\
& \cdot\left[e^{\alpha}\left(\left\|B_{1}\right\|+\left\|B_{\tau-1}\right\|+\left\|B_{\eta+1}\right\|+\cdots+\left\|B_{k}\right\|\right)\right. \\
& \left.+2(\underbrace{e^{\alpha_{\tau}}\left\|B_{\tau}\right\| \cos \beta_{\tau}+\cdots+e^{\alpha_{\eta}}\left\|B_{\eta}\right\| \cos \beta_{\eta}}_{(\eta-\tau+1) / 2})\right],
\end{aligned}
$$

where

$$
\begin{aligned}
& \lambda_{i}(P)=\left\{\alpha_{1}, \ldots, \alpha_{\tau-1}, \alpha_{\tau}+\beta_{\tau} j, \alpha_{\tau}-\beta_{\tau} j, \ldots, \alpha_{\eta}\right. \\
& \left.+\alpha_{\eta} j, \alpha_{\eta}-\beta_{\eta} j, \alpha_{\eta+1}, \ldots, \alpha_{k}\right\}, \\
& \alpha=\max \left\{\alpha_{1}, \ldots, \alpha_{k}\right\}, i=1, \ldots, \tau, \ldots, \eta, \ldots, k \leq n, \\
& B_{i}=\left[\begin{array}{cccccc}
1 & 1 & \frac{1}{2 !} & & \cdots & \frac{1}{\left(r_{i}-1\right) !} \\
& & \ddots & \ddots & & \\
& & \ddots & \ddots & \ddots & \\
& & & \ddots & \ddots & \frac{1}{2 !} \\
& & & & \ddots & 1 \\
& & & & 1
\end{array}\right],
\end{aligned}
$$

$r_{i}$ is the order of the same roots, and $\eta-\tau+1$ is an even number. Proof. The Taylor expansion of $\varphi(x)$ at $\lambda_{i}(P)$ can be written as

$$
\begin{aligned}
\varphi(x)= & \varphi\left(\lambda_{i}(P)\right)+\varphi^{\prime}\left(\lambda_{i}(P)\right)\left(x-\lambda_{i}(P)\right) \\
& +\frac{1}{2 !} \varphi^{\prime \prime}\left(\lambda_{i}(P)\right)\left(x-\lambda_{i}(P)\right)^{2}+\cdots \\
& +\frac{1}{n !} \varphi^{n}\left(\lambda_{i}(P)\right)\left(x-\lambda_{i}(P)\right)^{n}+\cdots
\end{aligned}
$$


Assume matrix $P$ is similar to a Jordan matrix $J$; that is,

$$
P \sim J=\left[\begin{array}{lll}
J_{1} & & \\
& \ddots & \\
& & J_{k}
\end{array}\right],
$$

where $i=1,2, \ldots, k \leq n$,

$$
J_{i}=\left[\begin{array}{cccc}
\lambda_{i}(P) & 1 & & \\
& \lambda_{i}(P) & \ddots & \\
& & \ddots & 1 \\
& & & \lambda_{i}(P)
\end{array}\right]_{r_{i} \times r_{i}}=\lambda_{i}(P) I+H
$$

with $H=J_{i}-\lambda_{i}(A) I$,

$$
\begin{gathered}
H=\left[\begin{array}{llll}
0 & 1 & & \\
& 0 & \ddots & \\
& & \ddots & 1 \\
& & & 0
\end{array}\right], \\
H^{2}=\left[\begin{array}{llll}
0 & 0 & 1 & \\
& 0 & \ddots & 1 \\
& & \ddots & 0 \\
& & & 0
\end{array}\right], \\
\vdots \\
H^{r_{i}-1}=\mathbf{0}_{r_{i} \times r_{i} .}
\end{gathered}
$$

Substituting $J_{i}=\lambda_{i}(P) I+H$ into (27) gives

$$
\begin{aligned}
\varphi\left(J_{i}\right)= & \varphi\left(\lambda_{i}(P)\right) I+\varphi^{\prime}\left(\lambda_{i}(P)\right)\left(J_{i}-\lambda_{i}(P) I\right) \\
& +\frac{1}{2 !} \varphi^{\prime \prime}\left(\lambda_{i}(P)\right)\left(J_{i}-\lambda_{i}(P) I\right)^{2}+\cdots \\
& +\frac{1}{n !} \varphi^{(n)}\left(\lambda_{i}(P)\right)\left(J_{i}-\lambda_{i}(P) I\right)^{n}+\cdots \\
= & \varphi\left(\lambda_{i}(P)\right) I+\varphi^{\prime}\left(\lambda_{i}(P)\right) H \\
& +\frac{1}{2 !} \varphi^{\prime \prime}\left(\lambda_{i}(P)\right) H^{2}+\cdots \\
& +\frac{1}{n !} \varphi^{(n)}\left(\lambda_{i}(P)\right) H^{n}+\cdots .
\end{aligned}
$$

Let $\varphi(x)=e^{x}$ and we have

$$
e^{J_{i}}=e^{\lambda_{i}(P)}\left[I+H+\frac{1}{2 !} H^{2}+\cdots\right]=e^{\lambda_{i}(P)} B_{i},
$$

where

$$
B_{i}=\left[\begin{array}{cccccc}
1 & 1 & \frac{1}{2 !} & & \cdots & \frac{1}{\left(r_{i}-1\right) !} \\
& \ddots & \ddots & \ddots & & \vdots \\
& & \ddots & \ddots & \ddots & \\
& & & \ddots & \ddots & \frac{1}{2 !} \\
& & & & \ddots & 1 \\
& & & & 1
\end{array}\right] .
$$

If there exists a matrix $Q$ such that $P=Q J Q^{-1}$, we have

$$
\begin{aligned}
\left\|e^{P}\right\| & =\left\|Q e^{J} Q^{-1}\right\| \leq\|Q\|\left\|e^{J}\right\|\left\|Q^{-1}\right\| \\
& =\|Q\|\left\|Q^{-1}\right\|\left(e^{\lambda_{1}}\left\|B_{1}\right\|+\cdots+e^{\lambda_{k}}\left\|B_{k}\right\|\right) .
\end{aligned}
$$

Assume $\lambda_{1}, \ldots, \lambda_{\tau-1}, \lambda_{\eta+1}, \ldots, \lambda_{k}$ are real roots, $\lambda_{\tau}, \ldots, \lambda_{\eta}$ are plurals, and $\lambda_{\tau}, \lambda_{\tau+1}$ is a pair of conjugate complex roots of $P$. It is obvious that $\lambda_{\eta-1}, \lambda_{\eta}$ is a pair of conjugate complex roots of $P$ and $\eta-\tau+1$ is an even number. Let

$$
\begin{array}{r}
\lambda_{\tau}=\alpha_{\tau}+\beta_{\tau} j, \\
\lambda_{\tau+1}=\alpha_{\tau}-\beta_{\tau} j
\end{array}
$$

then we have

$$
\begin{gathered}
e^{\lambda_{\tau}}\left\|B_{\tau}\right\|=e^{\alpha_{\tau}+\beta_{\tau} j}\left\|B_{\tau}\right\|=e^{\alpha_{\tau}} e^{\beta_{\tau} j}\left\|B_{\tau}\right\|, \\
e^{\lambda_{\tau+1}}\left\|B_{\tau+1}\right\|=e^{\alpha_{\tau}-\beta_{\tau} j}\left\|B_{\tau+1}\right\|=e^{\alpha_{\tau}} e^{-\beta_{\tau} j}\left\|B_{\tau+1}\right\| .
\end{gathered}
$$

According to the structure of $B_{i}$, we have $\left\|B_{\tau}\right\|=\left\|B_{\tau+1}\right\|$ and

$$
\begin{aligned}
e^{\lambda_{\tau}} & \left\|B_{\tau}\right\|+e^{\lambda_{\tau+1}}\left\|B_{\tau+1}\right\|=e^{\alpha_{\tau}}\left\|B_{\tau}\right\|\left(e^{\beta_{\tau} j}+e^{-\beta_{\tau} j}\right) \\
& =e^{\alpha_{\tau}}\left\|B_{\tau}\right\| \\
\cdot & {\left[\cos \beta_{\tau}+j \sin \beta_{\tau}+\cos \left(-\beta_{\tau}\right)+j \sin \left(-\beta_{\tau}\right)\right] } \\
= & 2 e^{\alpha_{\tau}}\left\|B_{\tau}\right\| \cos \beta_{\tau} .
\end{aligned}
$$

So $\left\|e^{P}\right\|$ can be written as

$$
\begin{aligned}
& \left\|e^{P}\right\|=\left\|Q e^{J} Q^{-1}\right\| \leq\|Q\|\left\|Q^{-1}\right\|\left[e^{\lambda_{1}}\|B\|_{1}+\cdots\right. \\
& +e^{\lambda_{\tau-1}}\left\|B_{\tau-1}\right\|+2 e^{\alpha_{\tau}}\left\|B_{\tau_{\tau}}\right\| \cos \beta+\cdots \\
& +2 e^{\alpha_{\eta-1}}\left\|B_{\eta-1}\right\| \cos \beta_{\eta-1}+e^{\lambda_{\eta+1}}\left\|B_{\eta+1}\right\|+\cdots \\
& \left.+e^{\lambda_{k}}\left\|B_{k}\right\|\right] \leq\|Q\|\left\|Q^{-1}\right\| \\
& {\left[e^{\alpha}\left(\left\|B_{1}\right\|+\left\|B_{\tau-1}\right\|+\left\|B_{\eta+1}\right\|+\cdots+\left\|B_{k}\right\|\right)\right.} \\
& \left.+2(\underbrace{e^{\alpha_{\tau}}\left\|B_{\tau}\right\| \cos \beta_{\tau}+\cdots+e^{\alpha_{\eta}}\left\|B_{\eta}\right\| \cos \beta_{\eta}}_{(\eta-\tau+1) / 2})\right]
\end{aligned}
$$

thus the proof is complete. 
Deduction 1. For the two order power systems with $P=A$, we have

$$
\left\|e^{A}\right\| \leq 1.618\|Q\|\left\|Q^{-1}\right\| e^{\alpha}
$$

if $A$ has two same real roots,

$$
\left\|e^{A}\right\| \leq 2\|Q\|\left\|Q^{-1}\right\| e^{\alpha}
$$

if $A$ has two different real roots, and

$$
\left\|e^{A}\right\| \leq 2 \cos (\beta)\|Q\|\left\|Q^{-1}\right\| e^{\alpha},
$$

if $A$ has a pair of conjugate complex roots, where $\alpha=$ $\max \left\{\operatorname{Re}\left(\lambda_{i}(A)\right)\right\}, i=1,2$.

Proof. Suppose $A$ has two same real roots; then $\alpha=\lambda_{1}=\lambda_{2}$ and

$$
A \sim J=\left[\begin{array}{ll}
\alpha & 1 \\
& \alpha
\end{array}\right]=\lambda I+H,
$$

where

$$
\begin{aligned}
I & =\left[\begin{array}{ll}
1 & 0 \\
0 & 1
\end{array}\right], \\
H & =\left[\begin{array}{ll}
0 & 1 \\
0 & 0
\end{array}\right], \\
H^{2} & =\mathbf{0}, \\
B_{1} & =\left[\begin{array}{ll}
1 & 1 \\
0 & 1
\end{array}\right], \\
\left\|B_{1}\right\| & =1.618 .
\end{aligned}
$$

According to Theorem 4, we have

$$
\begin{aligned}
\left\|e^{A}\right\| & =\left\|Q e^{J} Q^{-1}\right\| \leq\|Q\|\left\|Q^{-1}\right\|\left\|e^{J}\right\| \\
& \leq\|Q\|\left\|Q^{-1}\right\| e^{\alpha}\|B\|=1.618\|Q\|\left\|Q^{-1}\right\| e^{\alpha} .
\end{aligned}
$$

If $A$ has two real roots with $\lambda_{1} \neq \lambda_{2}$, choosing $\alpha=$ $\max \left\{\lambda_{1}, \lambda_{2}\right\}$, then we have

$$
A \sim J=\left[\begin{array}{ll}
\lambda_{1} & \\
& \lambda_{2}
\end{array}\right]
$$

There exists a matrix $Q$ such that $A=Q^{-1}$. So we have

$$
\begin{aligned}
\left\|e^{A}\right\| & =\left\|Q e^{J} Q^{-1}\right\| \leq\|Q\|\left\|Q^{-1}\right\|\left\|e^{J}\right\| \\
& \leq\|Q\|\left\|Q^{-1}\right\|\left(e^{\lambda_{1}}+e^{\lambda_{2}}\right) \leq 2\|Q\|\left\|Q^{-1}\right\| e^{\lambda} .
\end{aligned}
$$

If $A$ has a pair of conjugate complex roots, denote $\lambda_{i}(A)=$ $\alpha \pm \beta j$ and we have

$$
\begin{aligned}
& \left\|e^{A}\right\|=\left\|Q e^{J} Q^{-1}\right\| \leq\|Q\|\left\|Q^{-1}\right\|\left\|e^{J}\right\| \leq\|Q\|\left\|Q^{-1}\right\| \\
& \cdot\left(e^{\alpha+\beta j}+e^{\alpha-\beta j}\right)=\|Q\|\left\|Q^{-1}\right\| \\
& \cdot e^{\alpha}[\cos \beta+j \sin \beta+\cos (-\beta)+j \sin (-\beta)]=2 \\
& \cdot \cos \beta\|Q\|\left\|Q^{-1}\right\| e^{\alpha} ;
\end{aligned}
$$

thus the proof is complete.

Theorem 5. System (20) is interval mean stable if

$$
\lambda_{\max }\left[(\underline{A}+\bar{A})+(\underline{A}+\bar{A})^{T}\right]+2\|\bar{A}-\underline{A}\|<0,
$$

where $\lambda_{\max }\left[(\underline{A}+\bar{A})+(\underline{A}+\bar{A})^{T}\right]$ is the maximum eigenvalue of $\left[(\underline{A}+\bar{A})+(\underline{A}+\bar{A})^{T}\right]$, and

(1) if A has two same real roots, we have

$\lim _{t \rightarrow \infty} E\|x(t)\| \leq 1.618\|Q\|\left\|Q^{-1}\right\| \sqrt{\frac{2\|G\|^{2}+\left\|A^{\prime}\right\|^{2}}{-2 \alpha}} ;$

(2) if A has two different real roots, we have

$$
\lim _{t \rightarrow \infty} E\|x(t)\| \leq 2\|Q\|\left\|Q^{-1}\right\| \sqrt{\frac{2\|G\|^{2}+\left\|A^{\prime}\right\|^{2}}{-2 \alpha}} ;
$$

(3) if A has a pair of conjugate complex roots, we have

$$
\begin{aligned}
& \lim _{t \rightarrow \infty} E\|x(t)\| \\
& \quad \leq 2 \cos (\beta)\|Q\|\left\|Q^{-1}\right\| \sqrt{\frac{2\|G\|^{2}+\left\|A^{\prime}\right\|^{2}}{-2 \alpha}} .
\end{aligned}
$$

Proof. Consider the following system:

$$
d x(t)=A x(t) d t
$$

where $A$ is the same as the system matrix in (20).

Choosing a Lyapunov function $V=x^{T} x$, there exist positive constants $c_{1}$ and $c_{2}$ such that

$$
c_{1}\|x\|^{2} \leq V=x^{T} x \leq c_{2}\|x\|^{2},
$$


and $V$ has the infinitesimal upper bound and the infinite upper bound. Moreover, we have

$$
\begin{aligned}
& L V=2 x^{T} A x=2 x^{T} A^{*} x+2 x^{T} \Delta A x \\
& =x^{T}\left[(\underline{A}+\bar{A})+(\underline{A}+\bar{A})^{T}\right] x+2 x^{T} \Delta A x \\
& \leq \lambda_{\max }\left[(\underline{A}+\bar{A})+(\underline{A}+\bar{A})^{T}\right] x^{T} x \\
& +2\left\|x^{T}\right\|\|\Delta A x\| \\
& \leq \lambda_{\max }\left[(\underline{A}+\bar{A})+(\underline{A}+\bar{A})^{T}\right] x^{T} x+c_{3}\left\|x^{T}\right\|^{2} \\
& +\frac{1}{c_{3}}\|\Delta A x\|^{2} \\
& =\lambda_{\max }\left[(\underline{A}+\bar{A})+(\underline{A}+\bar{A})^{T}\right] x^{T} x+c_{3} x^{T} x \\
& +\frac{1}{c_{3}} x^{T} \Delta A^{T} \Delta A x \\
& \leq \lambda_{\max }\left[(\underline{A}+\bar{A})+(\underline{A}+\bar{A})^{T}\right] x^{T} x+c_{3} x^{T} x \\
& +\frac{1}{c_{3}}\|\Delta A\|^{2} x^{T} x \\
& \leq \lambda_{\max }\left[(\underline{A}+\bar{A})+(\underline{A}+\bar{A})^{T}\right] x^{T} x \\
& +\left(c_{3}+\frac{\|\Delta A\|^{2}}{c_{1} c_{3}}\right) x^{T} x \\
& \leq \lambda_{\max }\left[(\underline{A}+\bar{A})+(\underline{A}+\bar{A})^{T}\right] x^{T} x \\
& +\left(c_{3}+\frac{\|\widetilde{A}\|^{2}}{c_{1} c_{3}}\right) x^{T} x \\
& =\lambda_{\max }\left[(\underline{A}+\bar{A})+(\underline{A}+\bar{A})^{T}\right] x^{T} x \\
& +\left(c_{3}+\frac{\|(\bar{A}-\underline{A})\|^{2}}{4 c_{1} c_{3}}\right) x^{T} x,
\end{aligned}
$$

where $c_{3}>0$ is a constant. Let $c_{3}=(1 / 2)\|(\bar{A}-\underline{A})\|$ and $c_{1}=1$; we have

$L V$

$$
\leq\left\{\lambda_{\max }\left[(\underline{A}+\bar{A})+(\underline{A}+\bar{A})^{T}\right]+2\|\bar{A}-\underline{A}\|\right\} x^{T} x .
$$

Thus $L V<0$ and system (52) is stable under condition (48). Furthermore, we have $\alpha<0$.
Now consider the stochastic power system (20). The solution process of (20) can be expressed as [8]

$$
\begin{aligned}
x(t)= & e^{A t} x\left(t_{0}\right)+\int_{0}^{t} e^{A(t-\phi)} G d \phi \\
& +\int_{0}^{t} e^{A(t-\phi)} A^{\prime} d B(\phi) .
\end{aligned}
$$

Taking expectation of the both sides, we have

$$
\begin{aligned}
& E\left[x^{T}(t) x(t)\right]=E\left[\left(e^{A t} x_{0}\right)^{T} e^{A t} x_{0}\right] \\
& +E\left[\left(e^{A t} x_{0}\right)^{T} \int_{0}^{t} e^{A(t-\phi)} G d \phi\right] \\
& +E\left[\left(e^{A t} x_{0}\right)^{T} \int_{0}^{t} e^{A(t-\phi)} A^{\prime} d B(\phi)\right] \\
& +E\left[\left(\int_{0}^{t} e^{A(t-\phi)} G d \phi\right)^{T} e^{A t} x_{0}\right] \\
& +E\left[\left(\int_{0}^{t} e^{A(t-\phi)} G d \phi\right)^{T} \int_{0}^{t} e^{A(t-\phi)} G d \phi\right] \\
& +E\left[\left(\int_{0}^{t} e^{A(t-\phi)} G d \phi\right)^{T} \int_{0}^{t} e^{A(t-\phi)} A^{\prime} d B(\phi)\right] \\
& +E\left[\left(\int_{0}^{t} e^{A(t-\phi)} A^{\prime} d B(\phi)\right)^{T} \int_{0}^{t} e^{A(t-\phi)} A^{\prime} d B(\phi)\right] \\
& +E\left[\left(\int_{0}^{t} e^{A(t-\phi)} A^{\prime} d B(\phi)\right)^{T} e^{A t} x_{0}\right]
\end{aligned}
$$

Noticing that the expectation of the nonrandom variable is equal to itself and the expectation of the random variable is equal to 0 , we have

$$
\begin{gathered}
E\left[\left(e^{A t} x_{0}\right)^{T} e^{A t} x_{0}\right]=\left\|e^{A t} x_{0}\right\|^{2}, \\
E\left[\left(e^{A t} x_{0}\right)^{T} \int_{0}^{t} e^{A(t-\phi)} G d \phi\right] \\
=\left(e^{A t} x_{0}\right)^{T} \int_{0}^{t} e^{A(t-\phi)} G d \phi, \\
E\left[\left(\int_{0}^{t} e^{A(t-\phi)} G d \phi\right)^{T} e^{A t} x_{0}\right] \\
=\int_{0}^{t} G^{T} e^{A^{T}(t-\phi)} d \phi e^{A t} x_{0},
\end{gathered}
$$




$$
\begin{aligned}
& E\left[\left(\int_{0}^{t} e^{A(t-\phi)} G d \phi\right)^{T} \int_{0}^{t} e^{A(t-\phi)} G d \phi\right] \\
& =\int_{0}^{t} G^{T} e^{A^{T}(t-\phi)} d \phi \int_{0}^{t} e^{A(t-\phi)} G d \phi \\
& =\left\|\int_{0}^{t} e^{A(t-\phi)} G d \phi\right\|^{2} \\
& E\left[\left(\int_{0}^{t} e^{A(t-\phi)} A^{\prime} d B(\phi)\right)^{T} \int_{0}^{t} e^{A(t-\phi)} A^{\prime} d B(\phi)\right] \\
& =\int_{0}^{t} A^{\prime T} e^{A^{T}(t-\phi)} e^{A(t-\phi)} A^{\prime} d \phi=\int_{0}^{t}\left\|e^{A(t-\phi)} A^{\prime}\right\|^{2} d \phi, \\
& E\left[\left(e^{A t} x_{0}\right)^{T} \int_{0}^{t} e^{A(t-\phi)} A^{\prime} d B(\phi)\right]=0, \\
& E\left[\left(\int_{0}^{t} e^{A(t-\phi)} G d \phi\right)^{T} \int_{0}^{t} e^{A(t-\phi)} A^{\prime} d B(\phi)\right]=0, \\
& E\left[\left(\int_{0}^{t} e^{A(t-\phi)} A^{\prime} d B(\phi)\right)^{T} e^{A t} x_{0}\right]=0, \\
& E\left[\left(\int_{0}^{t} e^{A(t-\phi)} A^{\prime} d B(\phi)\right)^{T} \int_{0}^{t} e^{A(t-\phi)} G d \phi\right]=0 .
\end{aligned}
$$

Substituting (58) into (57), we can get

$$
\begin{aligned}
E\left[x^{T}(t) x(t)\right]= & \left\|e^{A t} x_{0}\right\|^{2}+\left(e^{A t} x_{0}\right)^{T} \int_{0}^{t} e^{A(t-\phi)} G d \phi \\
& +\int_{0}^{t} G^{T} e^{A^{T}(t-\phi)} d \phi e^{A t} x_{0} \\
& +\left\|\int_{0}^{t} e^{A(t-\phi)} G d \phi\right\|^{2} \\
& +\int_{0}^{t}\left\|e^{A(t-\phi)} A^{\prime}\right\|^{2} d \phi .
\end{aligned}
$$

According to Deduction 1, if $A$ has two same real roots, choose $c_{4}=1.618\|Q\|\left\|Q^{-1}\right\|$ and we have

$$
\left\|e^{A t} x_{0}\right\|^{2} \leq c_{4}^{2} e^{2 \alpha t}\left\|x_{0}\right\|^{2}
$$

Direct calculation shows that

$$
\begin{aligned}
& 2\left(e^{A t} x_{0}\right)^{T} \int_{0}^{t} e^{A(t-\phi)} G d \phi \\
& \quad \leq\left(e^{A t} x_{0}\right)^{T} e^{A t} x_{0}+\int_{0}^{t} G^{T} e^{A^{T}(t-\phi)} d \phi \int_{0}^{t} e^{A(t-\phi)} G d \phi
\end{aligned}
$$

$$
\begin{gathered}
=\left\|e^{A t} x_{0}\right\|^{2}+\left\|\int_{0}^{t} e^{A(t-\phi)} G d \phi\right\|^{2} \\
\leq\left\|e^{A t} x_{0}\right\|^{2}+\int_{0}^{t}\left\|e^{A(t-\phi)}\right\|^{2}\|G\|^{2} d \phi \\
\leq c_{4}^{2}\left[e^{2 \alpha t}\left\|x_{0}\right\|^{2}+\frac{\|G\|^{2}}{2 \alpha}\left(e^{2 \alpha t}-1\right)\right], \\
\int_{0}^{t} G^{T} e^{A^{T}(t-\phi)} d \phi e^{A t} x_{0} \\
\leq \frac{1}{2} c_{4}^{2}\left[e^{2 \alpha t}\left\|x_{0}\right\|^{2}+\frac{\|G\|^{2}}{2 \alpha}\left(e^{2 \alpha t}-1\right)\right], \\
\left\|\int_{0}^{t} e^{A(t-\phi)} G d \phi\right\|^{2} \leq c_{4}^{2} \frac{\|G\|^{2}}{2 \alpha}\left(e^{2 \alpha t}-1\right), \\
\int_{0}^{t}\left\|e^{A(t-\phi)} A^{\prime}\right\|^{2} d \phi \leq c_{4}^{2} \frac{\left\|A^{\prime}\right\|^{2}}{2 \alpha}\left(e^{2 \alpha t}-1\right) .
\end{gathered}
$$

Substituting (60)-(61) into (59) gives

$$
\begin{aligned}
& E\left[x^{T}(t) x(t)\right] \\
& \leq c_{4}^{2}\left[2 e^{2 \alpha t}\left\|x_{0}\right\|^{2}+\left(\frac{\|G\|^{2}}{\alpha}+\frac{\left\|A^{\prime}\right\|^{2}}{2 \alpha}\right)\left(e^{2 \alpha t}-1\right)\right] .
\end{aligned}
$$

According to Cauchy-Schwarz inequality

$$
(E\|x(t)\|)^{2} \leq E\|x(t)\|^{2}=E\left[x^{T}(t) x(t)\right],
$$

and noticing that

$$
E\|x(t)\| \leq \sqrt{E\left[x^{T}(t) x(t)\right]}
$$

we have

$$
\lim _{t \rightarrow \infty} E\|x(t)\| \leq 1.618\|Q\|\left\|Q^{-1}\right\| \sqrt{\frac{2\|G\|^{2}+\left\|A^{\prime}\right\|^{2}}{-2 \alpha}} .
$$

Similarly, if $A$ has two different real roots, we have

$$
\lim _{t \rightarrow \infty} E\|x(t)\| \leq 2\|Q\|\left\|Q^{-1}\right\| \sqrt{\frac{2\|G\|^{2}+\left\|A^{\prime}\right\|^{2}}{-2 \alpha}},
$$

and if $A$ has a pair of conjugate complex roots, we get

$$
\lim _{t \rightarrow \infty} E\|x(t)\|
$$

$$
\leq 2 \cos (\beta)\|Q\|\left\|Q^{-1}\right\| \sqrt{\frac{2\|G\|^{2}+\left\|A^{\prime}\right\|^{2}}{-2 \alpha}} .
$$

So there exists $\varepsilon_{1}>0$ such that

$$
\lim _{t \rightarrow \infty} E\|x(t)\|<\varepsilon_{1}
$$

and system (20) is interval mean stable. Thus the proof is complete. 
Theorem 6. System (20) is interval mean square stable, if

$$
\lambda_{\max }\left[(\underline{A}+\bar{A})+(\underline{A}+\bar{A})^{T}\right]+2\|\bar{A}-\underline{A}\|<0 .
$$

Moreover,

(1) if A has two the same real roots, we have

$$
\lim _{t \rightarrow \infty} E\|x(t)\|^{2} \leq 1.309\|Q\|^{2}\left\|Q^{-1}\right\|^{2} \frac{2\|G\|^{2}+\left\|A^{\prime}\right\|^{2}}{-\alpha}
$$

(2) if A has two different real roots, we have

$$
\lim _{t \rightarrow \infty} E\|x(t)\|^{2} \leq 2\|Q\|^{2}\left\|Q^{-1}\right\|^{2} \frac{2\|G\|^{2}+\left\|A^{\prime}\right\|^{2}}{-\alpha} ;
$$

(3) if A has a pair of conjugate complex roots, we get

$$
\begin{aligned}
& \lim _{t \rightarrow \infty} E\|x(t)\|^{2} \\
& \quad \leq 2 \cos ^{2}(\beta)\|Q\|^{2}\left\|Q^{-1}\right\|^{2} \frac{2\|G\|^{2}+\left\|A^{\prime}\right\|^{2}}{-\alpha} .
\end{aligned}
$$

Proof. The proof is similar to Theorem 5 and is omitted here.

Remark 7. Consider $n$-machine stochastic power systems described by

$$
\begin{aligned}
T_{t i} \frac{d \omega_{t i}}{d t}= & T_{w i}-K_{i} \theta_{w i}-D_{i}\left(\omega_{t i}-\omega_{r i}\right) \omega_{0} \\
& +\sigma_{1 i} W(t) \\
T_{g i} \frac{d \omega_{r i}}{d t}= & K_{i} \theta_{w i}+D_{i}\left(\omega_{t i}-\omega_{r i}\right) \omega_{0}-T_{e i}+\sigma_{2 i} W(t) .
\end{aligned}
$$

The system can be reformulated as

$$
d X(t)=\widehat{A} X(t) d t+\widehat{G} d t+\widehat{A}^{\prime} d B(t),
$$

where $X(t)$ is $2 n \times 1$ state matrix, $\widehat{A}$ is $2 n \times 2 n$ coefficient matrix, $\widehat{G}$ is $2 n \times 1$ gain matrix, and $\widehat{A}^{\prime}$ is $2 n \times 1$ random excitation matrix. From the proof procedure of Theorems 5 and 6 , we can see that similar results can be obtained for the interval mean and mean square stability of multimachine stochastic power systems.

\section{Simulation}

In this section, we simulate the power system to verify the effectiveness of the proposed results. The parameters of the system are chosen as $D \in$ [1.35 p.u., 1.65 p.u.], $T_{t}=10$ p.u., $T_{g}=2.5$ p.u., $\omega_{0}=1$ p.u., $s_{0}=0.08$ p.u., $r_{s}=0.003$ p.u., $x_{s}=$
0.125 p.u., $r_{r}=0.004$ p.u., $x_{r}=0.05$ p.u., $x_{m}=2.5$ p.u., and $U_{1}$ $=10.52$ p.u. and we can get $R=-7.23$,

$$
\begin{aligned}
\underline{A} & =\left[\begin{array}{ll}
-0.165 & -0.165 \\
-0.66 & -3.552
\end{array}\right], \\
\bar{A} & =\left[\begin{array}{ll}
-0.135 & -0.135 \\
-0.54 & -3.432
\end{array}\right], \\
\lambda_{\max } & {\left[(\underline{A}+\bar{A})+(\underline{A}+\bar{A})^{T}\right]+2\|\bar{A}-\underline{A}\|=-0.129 } \\
& <0 .
\end{aligned}
$$

According to Theorems 5 and 6, we know that the system is interval mean stability and interval mean square stability.

Choosing $D=1.45$ p.u. $\in$ [1.35 p.u., 1.65 p.u.], we can get

$$
A=\left[\begin{array}{cc}
-0.145 & -0.145 \\
-0.58 & -3.472
\end{array}\right] \text {, }
$$

$$
J=\left[\begin{array}{cc}
-0.1199 & 0 \\
0 & -0.34971
\end{array}\right] \text {, }
$$$$
Q=\left[\begin{array}{cc}
0.9854 & 0.0432 \\
-0.1705 & 0.9991
\end{array}\right]
$$

$$
Q^{-1}=\left[\begin{array}{cc}
1.0073 & -0.0436 \\
0.1719 & 0.9935
\end{array}\right]
$$

$$
G=\left[\begin{array}{ll}
-0.0116 & -0.0464
\end{array}\right]^{T},
$$

$A^{\prime}=\left[\begin{array}{ll}0.1 \sigma_{1} & -0.4 \sigma_{2}\end{array}\right]^{T}$,

$\|G\|^{2}=0.0022875$,

$\left\|A^{\prime}\right\|^{2}=0.01 \sigma_{1}^{2}+0.16 \sigma_{2}^{2}$,

$\|Q\|=1.0620$,

$\left\|Q^{-1}\right\|=1.0705$,

$\lambda_{1}(A)=-0.1199$,

$\lambda_{2}(A)=-0.34971$

so $\alpha=-0.1199$. According to Theorems 5 and 6 , the theoretical bounds of mean and mean square error with different $\sigma_{1}$ and $\sigma_{2}$ are shown in Table 1 when $t \rightarrow \infty$. The response of the system states with different $\sigma_{1}$ and $\sigma_{2}$ is shown in Figures 3-5. The bounds of mean and mean square error with different $\sigma_{1}$ and $\sigma_{2}$ are shown in Table 2, where $\left|x_{1}(t)\right|_{\max }$ and $\left|x_{2}(t)\right|_{\max }$ denote the absolute maximum values of stable states $\Delta \omega_{t}$ and $\Delta s$, respectively.

From Figure 3, we can see that the system states can converge fast to the stable operation under small excitation and the system is stable. When the random excitation intensity 
TABLE 1: The theoretical bounds of mean and mean square error.

\begin{tabular}{lccc}
\hline$\sigma_{1}$ & $\sigma_{2}$ & $E\|x(t)\|$ & $E\|x(t)\|^{2}$ \\
\hline 0.002 & 0.002 & 0.313327 & 0.098173 \\
0.003 & 0.003 & 0.313376 & 0.098204 \\
0.004 & 0.004 & 0.313416 & 0.098230 \\
0.005 & 0.005 & 0.313347 & 0.098263 \\
0.020 & 0.020 & 0.315643 & 0.099631 \\
0.030 & 0.030 & 0.318520 & 0.101454 \\
0.040 & 0.040 & 0.322503 & 0.104008 \\
0.050 & 0.050 & 0.327553 & 0.107291 \\
0.500 & 0.500 & 0.456192 & 0.208111 \\
1.000 & 1.000 & 0.928481 & 0.862078 \\
\hline
\end{tabular}
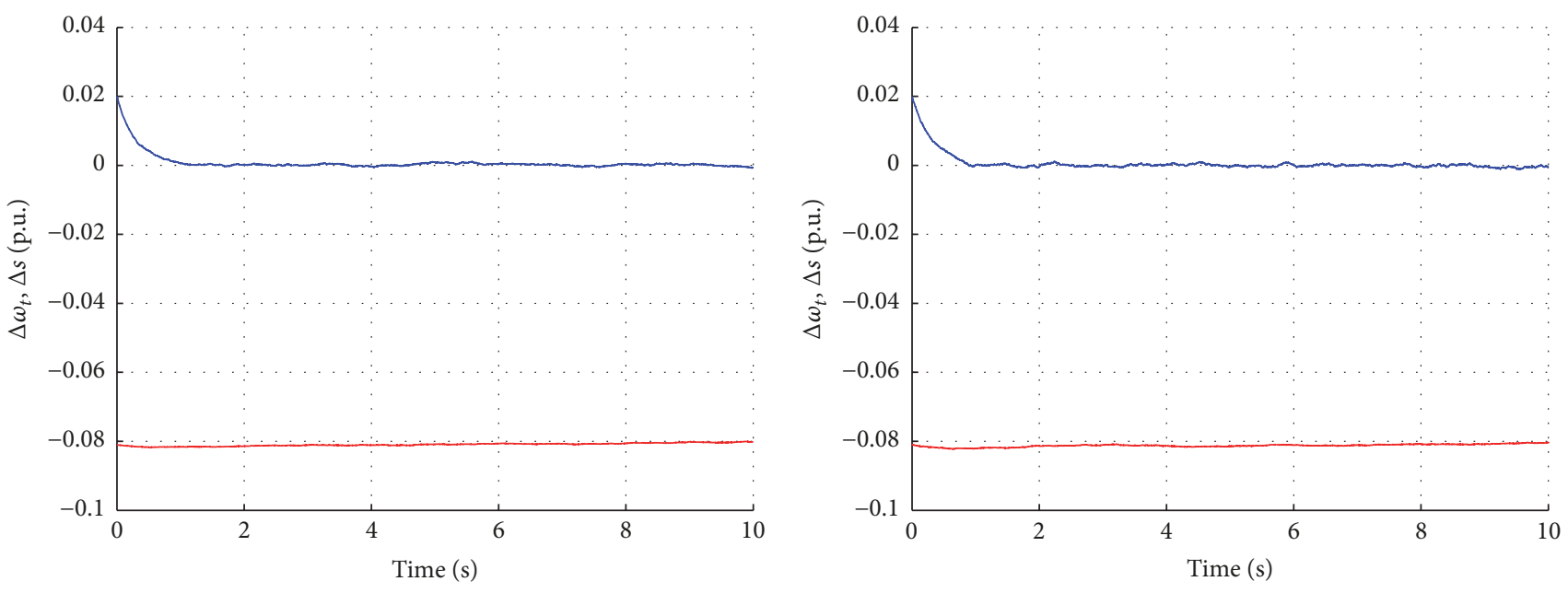

$-\Delta \omega_{t}$

$-\Delta \omega_{t}$

$-\Delta s$

$\left(\mathrm{a}_{1}\right)$

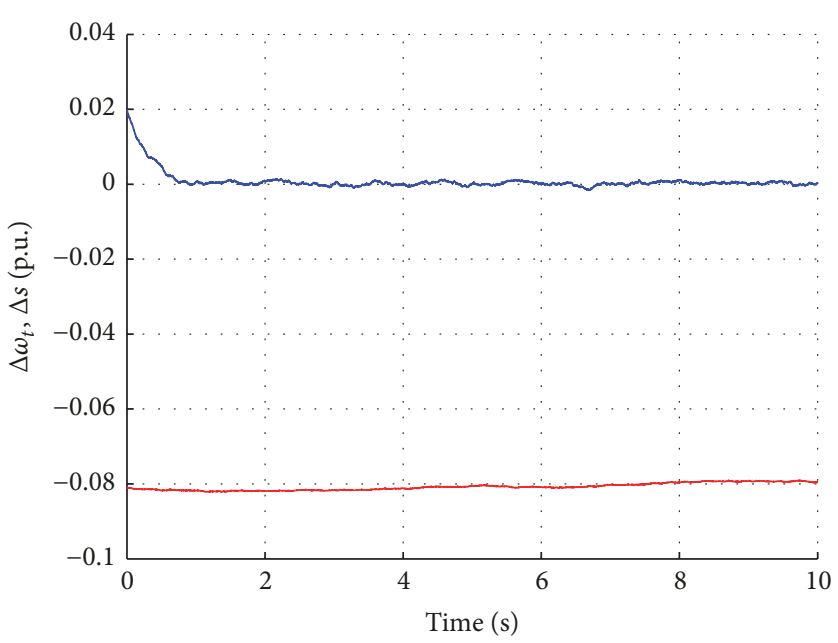

$\left(\mathrm{a}_{2}\right)$

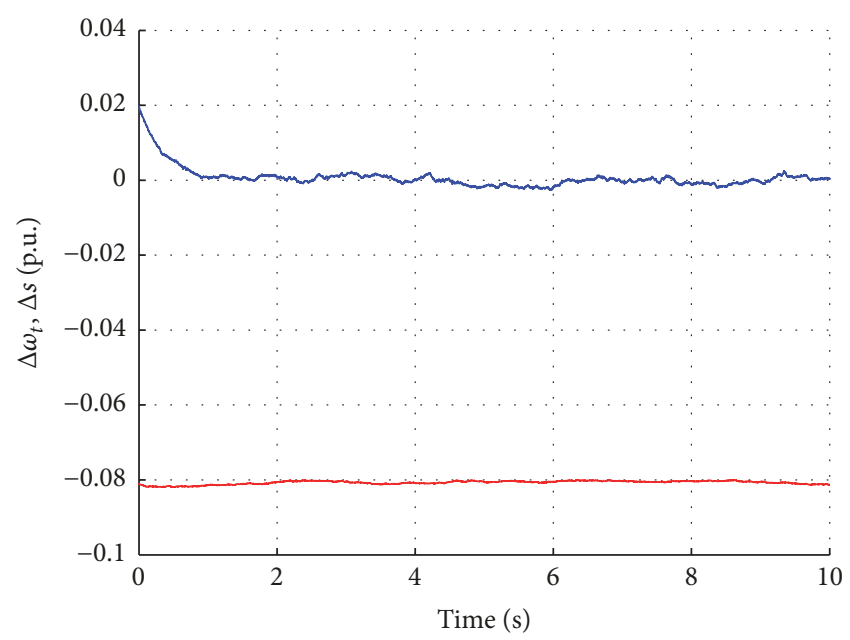

$-\Delta \omega_{t}$

$-\Delta \omega_{t}$

$-\Delta s$

$\left(a_{3}\right)$

$\left(a_{4}\right)$

Figure 3: Response of system with small random excitation. 
TABLE 2: The simulation bounds of mean and mean square error.

\begin{tabular}{lcccccc}
\hline $\begin{array}{l}\text { Corresponding } \\
\text { figure }\end{array}$ & $\sigma_{1}$ & $\sigma_{2}$ & $\left|x_{1}(t)\right|_{\max }$ & $\left|x_{2}(t)\right|_{\max }$ & $E\|x(t)\|$ & $E\|x(t)\|^{2}$ \\
\hline$\left(\mathrm{a}_{1}\right)$ & 0.002 & 0.002 & 0.080190 & 0.000792 & 0.080212 & 0.006431 \\
$\left(\mathrm{a}_{2}\right)$ & 0.003 & 0.003 & 0.080490 & 0.001105 & 0.080498 & 0.006480 \\
$\left(\mathrm{a}_{3}\right)$ & 0.004 & 0.004 & 0.080980 & 0.001229 & 0.080994 & 0.006560 \\
$\left(\mathrm{a}_{4}\right)$ & 0.005 & 0.005 & 0.081290 & 0.002478 & 0.081328 & 0.006614 \\
$\left(\mathrm{a}_{5}\right)$ & 0.020 & 0.020 & 0.082080 & 0.006803 & 0.082359 & 0.006783 \\
$\left(\mathrm{a}_{6}\right)$ & 0.030 & 0.030 & 0.086460 & 0.009684 & 0.087000 & 0.007569 \\
$\left(\mathrm{a}_{7}\right)$ & 0.040 & 0.040 & 0.087030 & 0.021220 & 0.089582 & 0.008025 \\
$\left(\mathrm{a}_{8}\right)$ & 0.050 & 0.050 & 0.093510 & 0.026570 & 0.097211 & 0.009450 \\
$\left(\mathrm{a}_{9}\right)$ & 0.500 & 0.500 & 0.193300 & 0.265300 & 0.328251 & 0.107749 \\
$\left(\mathrm{a}_{10}\right)$ & 1.000 & 1.000 & 0.377300 & 0.435100 & 0.575905 & 0.331667 \\
\hline
\end{tabular}

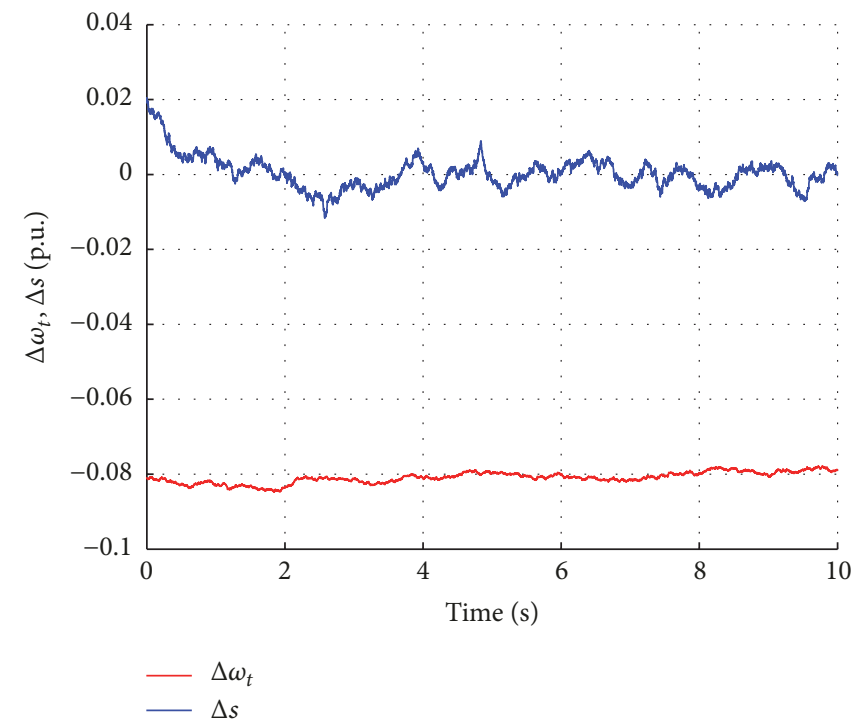

$\left(a_{5}\right)$

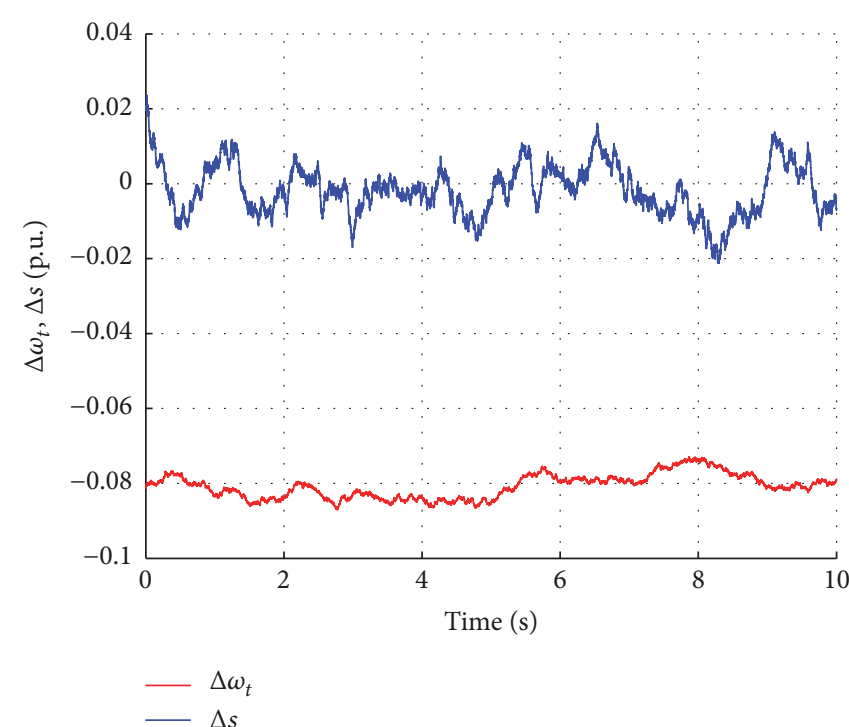

$\left(\mathrm{a}_{7}\right)$

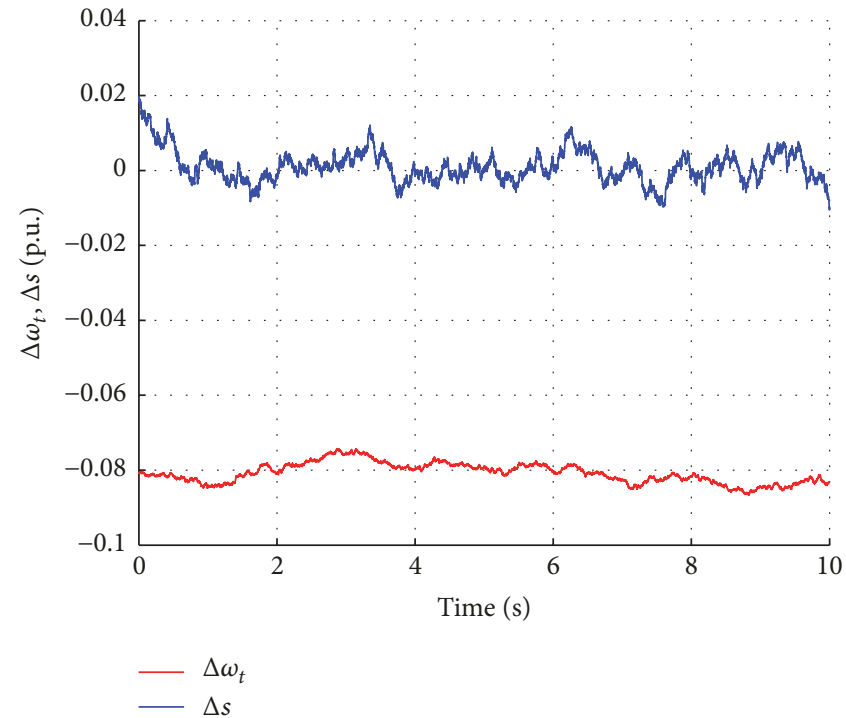

$\left(a_{6}\right)$

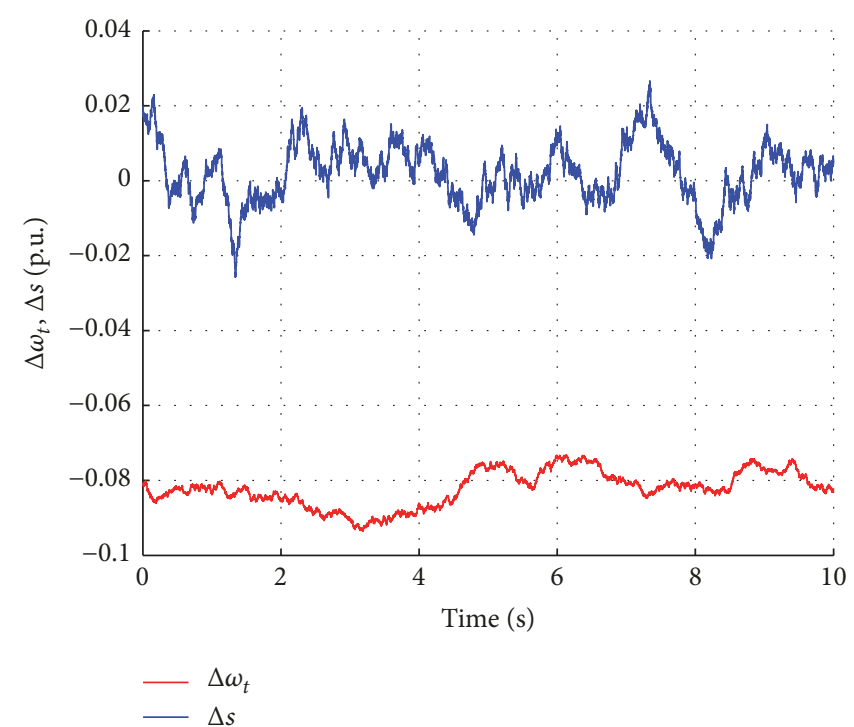

$\left(a_{8}\right)$

FIGURE 4: Response of system with large random excitation. 


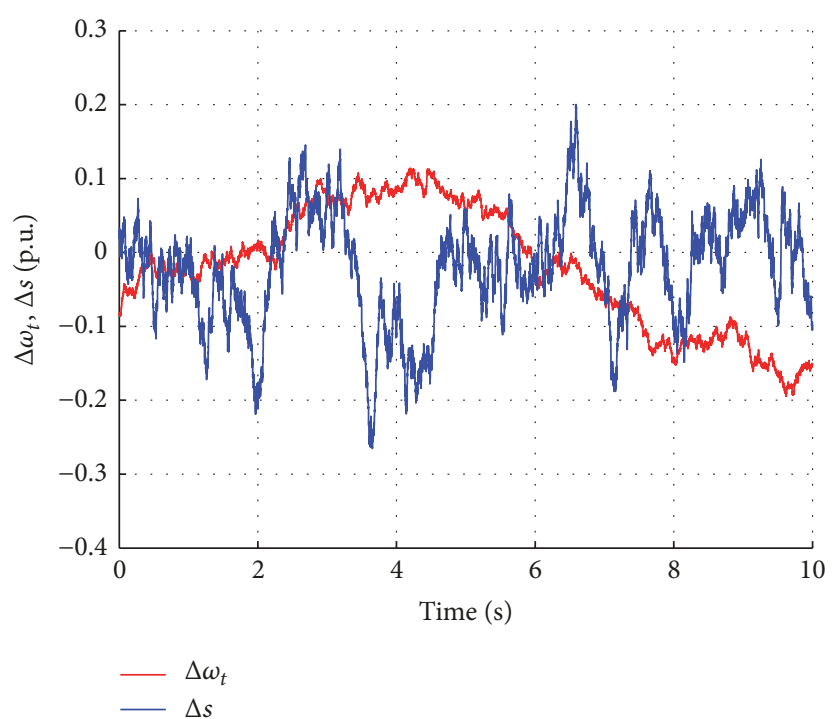

$\left(a_{9}\right)$

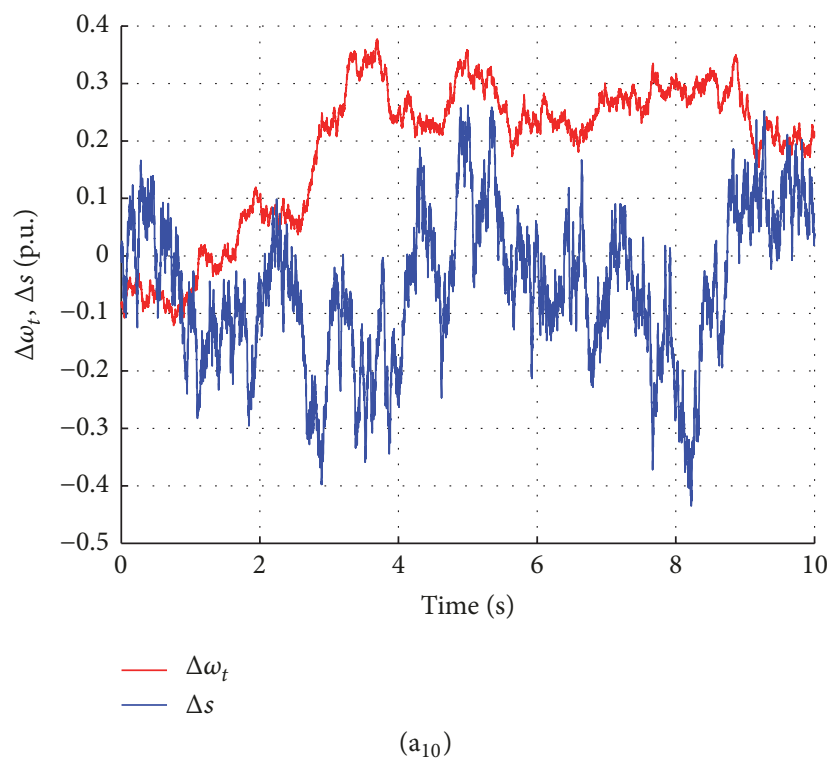

FIgURE 5: Response of system with larger random excitation.

increases, we can see from Figure 4 that the fluctuations of the system states increase compared to Figure 3. As shown in Figure 5, when we select a random excitation intensity largely, the system states fluctuate seriously and the system is unstable.

\section{Conclusions}

The paper established a stochastic interval dynamic model and analyzed the interval mean stability and interval mean square stability of power systems with asynchronous wind turbine generators. The relationship between the bounds of the mean and the mean square error of the system and the parameter interval range was discussed under random excitation. Theoretical analysis and simulation show that, for stochastic power systems with interval parameter uncertainties, the bounds of the mean and the mean square error are directly related to the random excitation. The power system will be unstable if the random excitation is large enough. Noticing that the mean square exponential stability analysis [23] and feedback controller design [24] for stochastic power systems are of great importance, in the future, we will carry out researches on the mean square exponential stability analysis of power systems with asynchronous wind turbine generators and design feedback controllers to improve the stochastic stability of the system under large random excitations.

\section{Conflicts of Interest}

The authors declare that they have no conflicts of interest. 


\section{Acknowledgments}

This work is supported by the National Natural Science Foundation of China (61473265, 61603345, and 61773351) and the Innovation Research Team of Science \& Technology of Henan Province (17IRTSTHN013).

\section{References}

[1] Y. Zhou, Y. Li, W. Liu, D. Yu, Z. Li, and J. Liu, "The stochastic response surface method for small-signal stability study of power system with probabilistic uncertainties in correlated photovoltaic and loads," IEEE Transactions on Power Systems, vol. 32, no. 6, pp. 4551-4559, 2017.

[2] J. Zhang, P. Ju, Y. Yu, and F. Wu, "Responses and stability of power system under small Gauss type random excitation," Science China Technological Sciences, vol. 55, no. 7, pp. 18731880, 2012.

[3] V. Humberto, K. Woifgang, V. Luis et al., "Stability region and radius in electric power systems under sustained random perturbations," International Journal of Electrical Power \& Energy Systems, vol. 73, pp. 725-733, 2015.

[4] J. Ma, Y. Qiu, Y. Li, and J. S. Thorp, "Stability analysis of power system with multiple operating conditions considering the stochastic characteristic of wind speed," IET Generation, Transmission \& Distribution, vol. 10, no. 4, pp. 1056-1066, 2016.

[5] H. Yue, G. Li, and M. Zhou, "A probabilistic approach to small signal stability analysis of power systems with correlated wind sources," Journal of Electrical Engineering \& Technology, vol. 8, no. 6, pp. 1605-1614, 2013.

[6] S. Q. Bu, W. Du, H. F. Wang, Z. Chen, L. Y. Xiao, and H. F. $\mathrm{Li}$, "Probabilistic analysis of small-signal stability of large-scale power systems as affected by penetration of wind generation," IEEE Transactions on Power Systems, vol. 27, no. 2, pp. 762-770, 2012.

[7] Q. Zhu, "Razumikhin-type theorem for stochastic functional differential equations with Lévy noise and Markov switching," International Journal of Control, vol. 90, no. 8, pp. 1703-1712, 2017.

[8] X. Mao, Stochastic Differential Equations and Applications, Horwood Press, Chichester, UK, 2nd edition, 1997.

[9] H. Wang and Q. Zhu, "Finite-time stabilization of high-order stochastic nonlinear systems in strict-feedback form," Automatica, vol. 54, pp. 284-291, 2015.

[10] B. Wang and Q. X. Zhu, "Stability analysis of Markov switched stochastic differential equations with both stable and unstable subsystems," Systems \& Control Letters, vol. 105, pp. 55-61, 2017.

[11] V. Humberto, V. Luis, K. Woifgang et al., "Stability of linear stochastic systems via Lyapunov exponents and applications to power systems," Applied Mathematics \& Computation, vol. 218, no. 22, pp. 11021-11032, 2012.

[12] Y. Sun, X. Zhao, N. Li, Z. Wei, and G. Sun, "Robust stochastic stability of power system with time-varying delay under Gaussian random perturbations," Neurocomputing, vol. 162, pp. 1-8, 2015.

[13] Y. Xu, M. Yin, Z. Dong, R. Zhang, D. J. Hill, and Y. Zhang, "Robust dispatch of high wind power-penetrated power systems against transient instability," IEEE Transactions on Power Systems, vol. 99, pp. 1-1, 2017.

[14] Q. X. Zhu and Q. Y. Zhang, "pth moment exponential stabilisation of hybrid stochastic differential equations by feedback controls based on discrete-time state observations with a time delay," IET Control Theory \& Applications, vol. 11, no. 12, pp. 1992-2003, 2017.

[15] Q. Zhu, "Asymptotic stability in the pth moment for stochastic differential equations with Lévy noise," Journal of Mathematical Analysis and Applications, vol. 416, no. 1, pp. 126-142, 2014.

[16] B. Yuan, M. Zhou, G. Li, and X.-P. Zhang, "Stochastic smallsignal stability of power systems with wind power generation," IEEE Transactions on Power Systems, vol. 30, no. 4, pp. 16801689, 2015.

[17] Z. H. Lu, W. X. Zhao, and D. Xie, "P-moment stability of power system under small Gauss type random excitation," Chaos Solitons Fractals the Interdisciplinary Journal of Nonlinear Science Nonequilibrium Complex Phenomena, vol. 81, pp. 30-37, 2015.

[18] Z. N. Wei, Q. Yan, G. Q. Sun et al., "Power system interval linear state estimation considering network parameter uncertainty," Journal of the Acm, vol. 2, no. 2, pp. 99-110, 2015.

[19] C. Rakpenthai, S. Uatrongjit, and S. Premrudeeprechacharn, "State estimation of power system considering network parameter uncertainty based on parametric interval linear systems," IEEE Transactions on Power Systems, vol. 27, no. 1, pp. 305-313, 2012.

[20] G. Q. Li. Power System, Power System Trans.ient Analysis Inequality, Water Power Press, Beijing, China, 1985.

[21] S. G. Wang, M. X. Wu, and Z. Z. Jia, Matrix Inequality, Science Press, Beijing, China, 2006, Matrix Inequality, Science Press.

[22] Z. E. Ma and Y. C. Zhou, Qualitative and Stability for Ordinary Differential Equations, Science Press, Beijing, China, 2005.

[23] Q. Zhu, S. Song, and T. Tang, "Mean square exponential stability of stochastic nonlinear delay systems," International Journal of Control, vol. 90, no. 11, pp. 2384-2393, 2017.

[24] H. Wang and Q. X. Zhu, "Global Stabilization of Stochastic Nonlinear Systems via $\mathrm{Cl}$ and C8 Controllers," IEEE Transactions on Automatic Control, vol. 62, no. 11, pp. 5880-5887, 2017. 


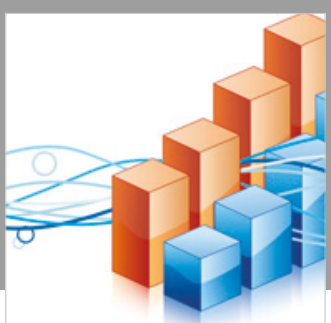

Advances in

Operations Research

\section{-n-m}
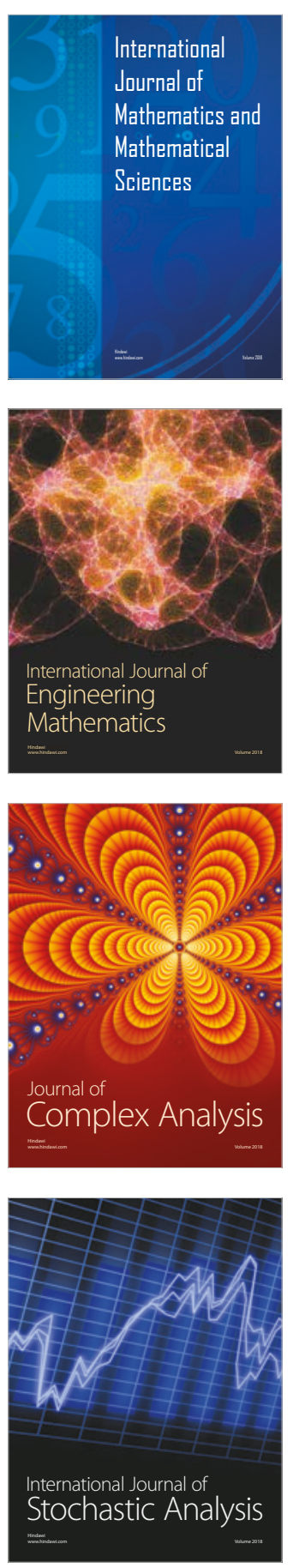
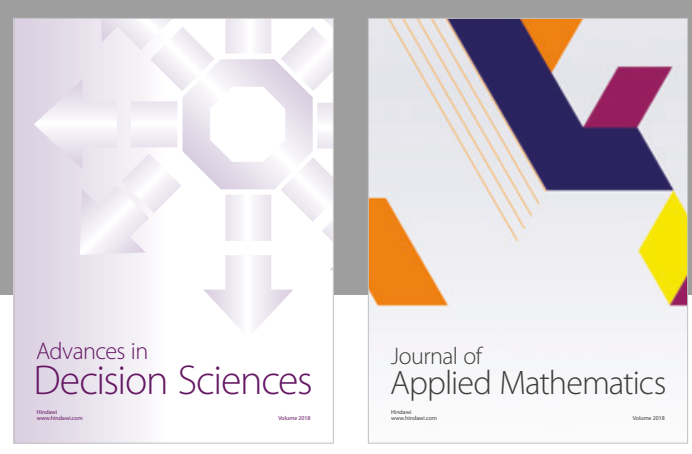

Journal of

Applied Mathematics
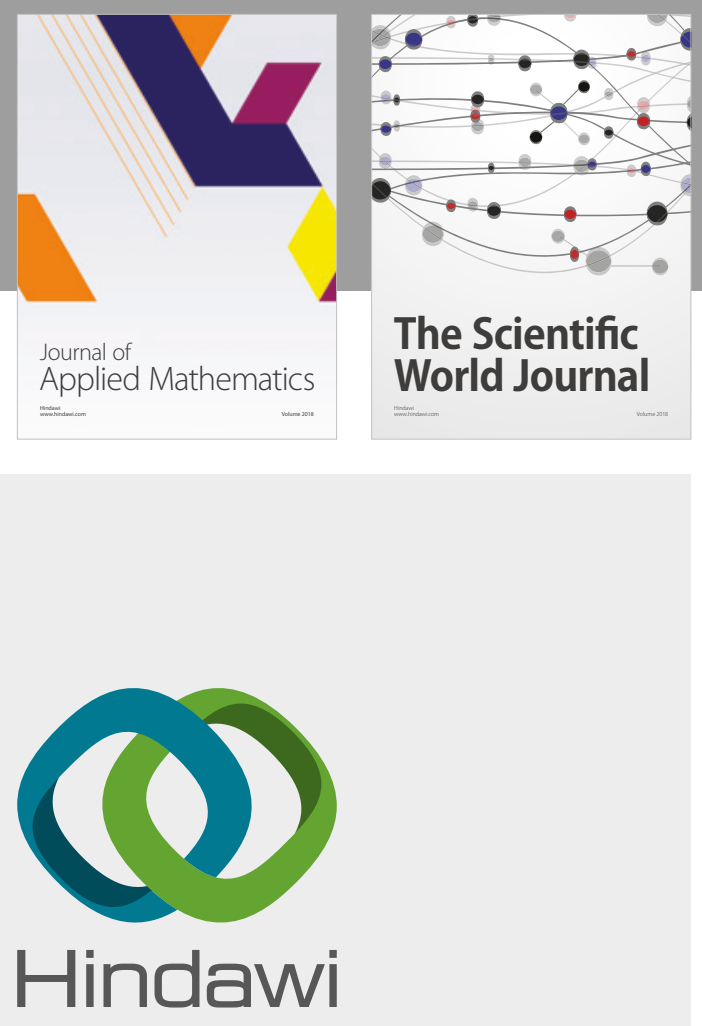

Submit your manuscripts at

www.hindawi.com

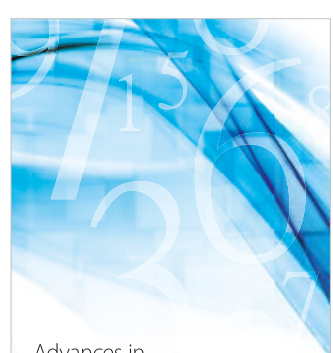

Advances in
Numerical Analysis
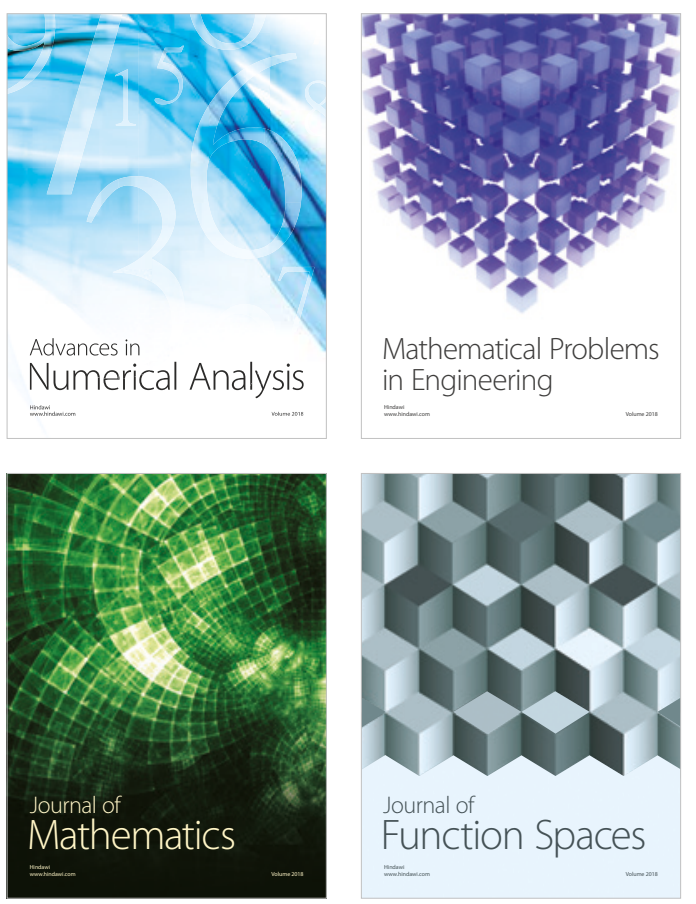

Mathematical Problems in Engineering

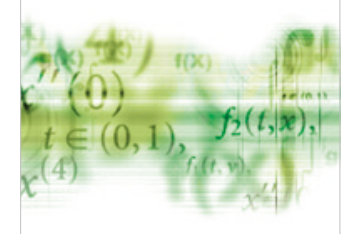

International Journal of

Differential Equations

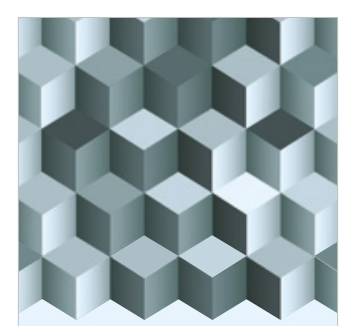

Journal of

Function Spaces

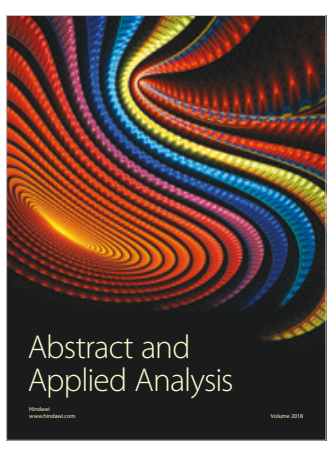

The Scientific

World Journal

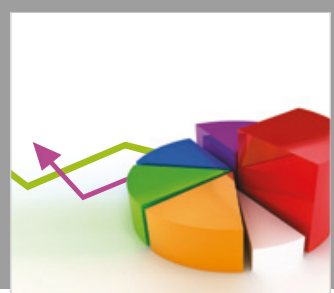

Journal of

Probability and Statistics
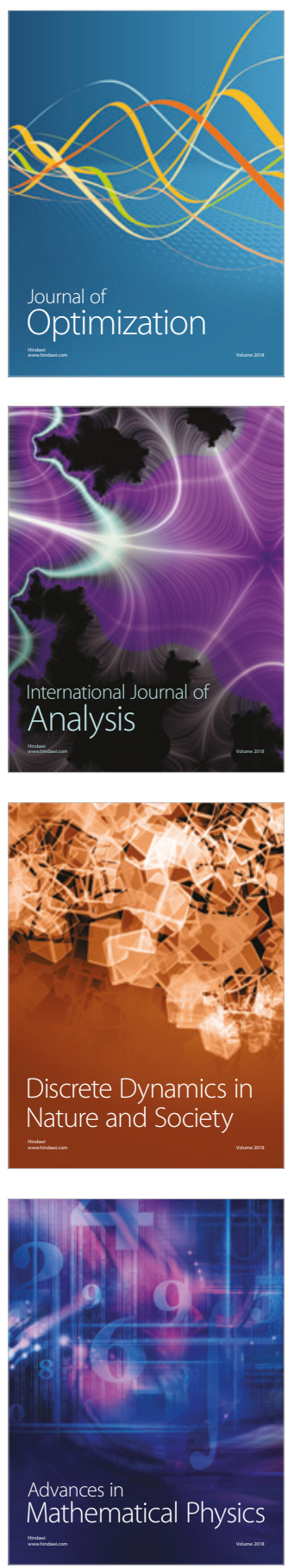